\title{
$1 \quad$ Is Placing Prophylactic Dural Tenting Sutures a Dogma?
}

3 Umit Eroglu, MD, ${ }^{1}$ Murat Zaimoğlu, MD, ${ }^{1}$ Emre Yağız Sayacı, MD, ${ }^{1}$ Hasan Caglar Ugur, MD,

4 PhD,${ }^{1}$ Ayhan Attar, MD,${ }^{1}$ Gokmen Kahilogullari, MD, PhD,${ }^{1}$ Melih Bozkurt, MD, ${ }^{1}$ Mustafa

5 Ağahan Ünlü, MD, ${ }^{1}$ Onur Özgüral, MD, ${ }^{1}$ İhsan Doğan, MD, ${ }^{1}$ Kutsal Devrim Seçinti, MD ${ }^{2}$ Bilal

6 Abbasoğlu, MD, ${ }^{1}$ Koral Erdoğan, MD, ${ }^{1}$ Elif Gökalp, MD, ${ }^{1}$ Fatih Yakar, MD ${ }^{3}$ Yusuf Şükrü

7 Çağlar, MD, ${ }^{1}$ and Aaron Cohen-Gadol, MD, MSc, MBA ${ }^{4,5}$

$9 \quad{ }^{1}$ Faculty of Medicine, Department of Neurosurgery, Ankara University, Ankara, Turkey;

$10{ }^{2}$ Faculty of Medicine, Department of Neurosurgery, Kahramanmaraş Sütçü İmam University,

11 Maras, Turkey; ${ }^{3}$ Faculty of Medicine, Department of Neurosurgery, Pamukkale University,

12 Denizli, Turkey; ${ }^{4}$ The Neurosurgical Atlas, Carmel, Indiana; and ${ }^{5}$ Department of Neurological

13 Surgery, Indiana University School of Medicine, Indianapolis, Indiana

15 Correspondence: Aaron Cohen-Gadol, MD, MSc, MBA, Department of Neurological Surgery,

16 Indiana University, 355 W 16th Street, Suite 5100, Indianapolis, IN 46202; cohen@nsatlas.com

18 Short Title: Prophylactic Dural Tenting Suturing

20 Key Words: epidural hematoma; dural tenting suture; complication; craniotomy; subdural

21 hematoma; iatrogenic

23 Conflict of interest: None.

25 Funding: This research did not receive any specific grant from funding agencies in the public, 26 commercial, or not-for-profit sectors. 
1 Is Placing Prophylactic Dural Tenting Sutures a Dogma? 
ABSTRACT

3 Objective: In this study, we investigated if and when dural tenting sutures are necessary

4 during craniotomy.

Methods: Results from 437 patients aged 18 to 91 years (average, 43.5 years) who underwent

7 supratentorial craniotomy between 2014 and 2019 were evaluated. The patients were

8 categorized into 1 of 3 groups, patients who had at least 3 prophylactic dural tenting sutures

9 placed before opening of the dura (group 1), at least 3 dural tenting sutures placed after surgery was completed, during closure (group 2), or no dural tenting sutures (group 3

11 [control]). All such sutures in groups 1 and 2 were placed in the circumference of the

12 craniotomy and dural junction. No central dural tenting sutures were placed in any of the 13 patients.

15 Results: Among the 437 patients, 344 underwent surgery for the first time and 93 were undergoing a second surgery. Cranial computed tomography imaging was performed for each

17 patient 1 hour, 3 days, and 1 month after surgery. In group 1, 3 patients had a cerebral cortex

18 contusion and 2 patients had acute subdural hematoma after the sutures were placed. In

19 groups 2 and 3, none of the patients had a cerebral cortex contusion or acute subdural

20 hematoma. Fewer complications were observed when dural tenting sutures were placed

21 during postsurgical closure.

23 Conclusion: Placing dural tenting sutures is an important technique for ensuring hemostasis.

24 However, when not needed, they seem to cause inadvertent complications. As our results 25 suggest, knowing when and where to use them is equally important. 


\section{INTRODUCTION}

Craniotomy is an indispensable neurosurgical procedure, and many surgical approaches and craniotomy types exist. However, important complications related to craniotomy closure and the postcraniotomy period can occur. The use of dural tenting sutures between the dura and the galea or subaponeurotic tissue to prevent postoperative epidural hematoma (EDH) was first described by Walter Edward Dandy in $1932 .{ }^{1}$ Horsley $^{2,3}$ and Cushing ${ }^{4,5}$ also frequently used electrocoagulation in neurosurgical operations to prevent postoperative EDH.

However, the need for dural tenting suture placement, which has continued in neurosurgery for the past 2 decades, has begun to be questioned in light of modern hemostasis, hemostatic agents, and anesthesia. Are prophylactic dural tenting sutures really necessary, and if so, at which stage of the surgery should they be placed? In this study, 437 patients who underwent craniotomy between May 2016 and February 2021 at the Ankara University Department of Neurosurgery were evaluated. Complications such as the presence and number of EDHs, emerging new neurological deterioration, cerebrospinal fluid (CSF) leak, cortex tissue damage, and dural tenting suture-related complications, such as subdural hygroma and foreign-body reaction, were examined.

\section{MATERIALS AND METHODS}

\section{Patient Information}

This prospective study included 437 patients who were older than 18 years. All patients participating in the study underwent a supratentorial craniotomy between May 2016 and February 2021 at the Ankara University Department of Neurosurgery. All intra-axial, extraaxial, and vascular pathologies were included in the study. Patients who underwent a posterior fossa craniotomy, craniotomy $<2 \mathrm{~cm}$ in diameter, external ventricular drainage placement 
surgery, endoscopic intervention, or a biopsy procedure, emergency trauma patients with multiple head bone fractures, patients who had an epidural and/or subdural external drain placed during surgery, patients who underwent CSF drainage (eg, lumbar puncture) after surgery, and patients using an anticoagulant(s) were excluded.

\section{Surgical Technique}

4-0 silk sutures were used in all patients. The patients included in the study were divided into 3 groups. Group 1 patients had at least 3 prophylactic dural tenting sutures placed before the dura was opened. The dura was grasped with Adson forceps, elevated upward with a dural needle, and grasped again with the forceps for placement of the tenting sutures to avoid possible cortex damage. In group 2 patients, the dura was opened, and after the surgery was completed, at least 3 dural tenting sutures were placed with the use of an operating microscope, which allowed viewing of the distance between the dura and the cortex. Dural tenting sutures were not used in any patients in group 3 (the control group).

Bone wax was used on the bone margins and oxidized regenerated cellulose was used at the bone-dura junction in all patients. Dural hemostasis was performed in all patients. In the patient groups receiving dural tenting sutures, the sutures were hung from their closest location to the bone, thereby causing the dura to adhere more strongly to the bone.

\section{Statistical Analysis}

All statistical analyses were performed using SPSS for Windows version 11.5 (SPSS, Inc, Chicago, IL). Descriptive statics are expressed as number (percentage) for categorical variables. Chi-square and Fisher exact tests were used to analyze the relationship between 2 categorical variables. A $P$ value of 0.05 was considered statistically significant. 


\section{Ethical Approval}

78

This study was performed with approval from the Ankara University Ethics Committee (number 11-64-20).

\section{RESULTS}

All 437 patients underwent surgery performed at the Ankara University Department of Neurosurgery. The study consisted of 236 male and 201 female patients aged 18 to 91 years (average age, 43.5 years). Primary surgery was performed in 344 patients and secondary surgery in 93 patients. The majority of the patients required surgery because of a tumor (Table 1). Groups 1, 2, and 3 included 146, 146, and 145 patients, respectively. As the statistical analysis suggested and confirmed, the preoperative surgical information of the patients constitutes a heterogenous group, and the data apply to real-time circumstances. Cranial computed tomography (CT) imaging was performed in all patients at 1 hour, 3 days, and 1 month after surgery.

In group 1, 3 of the patients had a cerebral cortex contusion, and 2 patients had acute subdural hematoma (Figure 1). In postoperative cranial CT images, an epidural collection smaller than or equal to $3 \mathrm{~mm}$ was detected in 6 patients, and an epidural collection larger than $3 \mathrm{~mm}$ was detected in 3 patients.

In group 1, 1 patient who experienced neurologic deterioration that was caused by acute subdural hematoma required reoperation. CSF was accumulated under the skin in 7 patients and was treated with palliative measures. The cause of acute subdural hematoma in 2 patients was venous (1 patient) and arterial (1 patient) injury between the dura and the pia. 
102 In group 2, no patient had cortical damage or acute subdural hematoma as a result of the dural tenting sutures. Epidural collections of $\leq 3 \mathrm{~mm}$ and $>3 \mathrm{~mm}$ were detected in 5 and 4 patients, respectively. None of the patients required reoperation because of an epidural collection. CSF accumulated under the skin in 5 patients and was treated with palliative measures.

106

107 In group 3, none of the patients had cerebral cortex contusion or acute subdural hematoma.

108 Epidural collections of $\leq 3$ and $>3 \mathrm{~mm}$ were observed in 7 and 3 patients, respectively. CSF

109 accumulated under the skin in 5 patients and was treated with palliative measures.

111 As for statistical analysis, we examined whether a difference between the groups existed in

112 terms of postoperative complications, and no significant difference was found for any of the 113 variables between any of the groups $(P>0.05)$.

114

115 Subdural hygroma, detected by control CT imaging 1 month after surgery, was more frequent

116 in group 1 than in groups 2 and 3, but this difference was not statistically significant (8 versus

1175 and 4 cases, respectively) (Table 2). Craniotomy sizes varied between 2 and $15 \mathrm{~cm}$. We

118 found no difference in postoperative complications according to craniotomy size among the 3 119 groups.

\section{DISCUSSION}

122 EDHs are one of the most important causes of acute and late morbidity and death after

123 craniotomy. ${ }^{1}$ Horsley, ${ }^{6,7}$ Cushing, ${ }^{8,9}$ and Poppen ${ }^{10}$ developed many methods for avoiding

124 these complications. 
Insufficient control of patient blood pressure after the induction of anesthesia and inadequate

127 imaging methods resulted in the routine neurosurgical use of dural tenting sutures to prevent

128 EDH. Prophylactic dural tenting sutures are still used routinely today to prevent possible late

129 and/or acute complications. In recent studies, the occurrences of an EDH of $>3 \mathrm{~mm}$ after

130 craniotomy ranged from $0.2 \%$ to $2.6 \% .^{11-15}$

131

132 The numbers of patients who had an EDH of $>3 \mathrm{~mm}$ were not significantly different among

133 the groups. The use of prophylactic epidural tenting sutures did not make any difference in the

134 prevention of epidural collection. Except for in group 1, cortex injury and subdural hematoma

135 were not observed. The obvious reason for this difference was the lack of space between the

136 brain and dura as a result of increased intracranial pressure. Subdural hygromas and

137 hematomas developing after surgery are an important neurosurgical entity. ${ }^{16}$

139 An expected but striking result was the greater rate of subdural hygromas in group 1. Even in

140 very skillful and experienced hands, placing dural tenting sutures without actually visualizing

141 the underlying cortex can result in cortical or dural bridging vein damage. These data support

142 the idea that visualizing underlying tissue before dural tenting suture placement is a safer and

143 a more reliable approach regardless of the surgeon-related factors. Subdural hygromas are

144 more common in elderly patients and in patients who undergo large-volume lesion excision.

145 This gap, which is decompressed after surgery and is greater than the normal space created

146 between the excessively collapsed brain tissue and the dura, manifests itself as a hygroma in

147 the late period. If the brain tissue does not expand sufficiently and this gap increases, a

148 subdural hygroma that requires surgery may develop. More important, however, is that an

149 increase in this gap may be a risk factor for the development of late dural bridging vein

150 hemorrhage. Tumor size and location and degree of decompression (CSF drainage through 
151 the cisterna intraoperatively) are also factors changing this gap volume and can contribute to

152 the formation of subdural hygromas. We did not assess these variables, and this is a limitation

153 of our study.

155 In group 2 patients, dural sutures were placed using an operative microscope after lesion

156 excision. A safe space between the dura and the cortex was observed with the microscope.

157 Bridging veins between the cortex and the dura, which cannot be seen before opening the

158 dura, were well visualized. The safe passage of the dural needle was observed under the

159 microscope (Figure 2), which enabled safer placement of the dural tenting sutures (Figure 3).

161 Oxidized regenerated cellulose and bone wax were used in all 3 groups. Oxidized regenerated

162 cellulose was not applied between the dura and the bone, because this procedure can cause unnecessary dural dissection and epidural collection.

165 It is important to perform surgery while the patient has normotensive blood pressure during

166 anesthesia. The Valsalva maneuver is frequently performed so that possible bleeding can be

167 detected for hemostasis. However, if traditional prophylactic dural tenting sutures are to be

168 used, the Valsalva maneuver should be performed after watertight dural closure.

170 The complication rates were similar to those reported in the literature. Pial fistulas, foreign-

171 body reactions, and CSF leakage caused by dural sutures were important complications

172 reported in the literature. However, no patient in this series encountered any of these

173 complications. ${ }^{17,18} \mathrm{CSF}$ accumulation resolved with palliative measures in all groups, and did

174 not progress to CSF leakage. 
176 Ninety-three patients in this study who had previously had a craniotomy underwent another

177 procedure. These patients underwent their surgery at least 6 months before the second

178 operation. In these patients, dural tenting sutures were used in neither the first nor the second

179 operation. Because cranial epidural fibrosis is formed between the dura and cranial bone after

180 the first surgery, dural tenting sutures were not needed.

181

182 Very few studies regarding the necessity for dural tenting sutures exist in the literature. ${ }^{19-22}$ In

183 those few studies, the need to use dural tenting sutures was questioned. However, for the first

184 time, our study included consideration of the timing for placement of the dural tenting sutures.

186 Conclusions

187 Placing dural tenting sutures is an important surgical technique for hemostasis. If these

188 sutures are placed before the dura is opened, they seem to cause unnecessary complications.

189 However, if they are placed after the dura is opened, a lower number of complications is seen.

190 When no dural tenting sutures were used, no complications after surgery were reported. This

191 result shows that placing tenting sutures might not be necessary; however, there is not enough

192 evidence to support this claim. More research on this topic is needed.

193

194 As this study shows for the first time, the best time to place dural tenting sutures is during

195 postsurgical closure. In patients who underwent a second operation, epidural tenting sutures

196 were not necessary, because epidural fibrosis was present and the dura was strictly adherent to

197 overlying bone structures.

198 
199 Dural tenting sutures may or may not be used by neurosurgeons, and they are used most

200 commonly by choice. The questions of whether to use these sutures, when to place them, and 201 how to place them require more study and research data to be evaluated further.

202

\section{ACKNOWLEDGMENT}

204 The authors sincerely appreciate the support of the Stead Family Endowed Chair in creation 205 of this work. 


\section{REFERENCES}

207 1. Dandy WE. Surgery of the brain. In: Lewis' Practice of Surgery, Vol 12. Hagerstown, 208 MD: WF Prior; 1945:144-162.

209 2. Horsley V. Antiseptic wax. Br Med J. 1892;1(1639):1165.

2103 . Horsley V. Remarks on ten consecutive cases of operations upon the brain and cranial 211 cavity to illustrate the details and safety of the method employed. $\mathrm{Br} \mathrm{Med} J$. 1887;1(1373):863-865.

4. Cushing $\mathrm{H}$. The control of bleeding in operations for brain tumors: with the description of silver "clips" for the occlusion of vessels inaccessible to the ligature. Ann Surg. 1911;54:1-19.

5. Cushing H. Electro-surgery as an aid to the removal of intracranial tumors. Surg Gynecol Obstet. 1928;47:751-784.

6. Horsley V. Antiseptic wax. Br Med J. 1892:1:1165.

7. Horsley V. Remarks on ten consecutive cases of operations upon the brain and cranial cavity to illustrate the details and safety of the method employed. Br Med J. 1:863-865, 1887.

8. Cushing H. The control of bleeding in operations for brain tumors. With the description of silver "clips" for the occlusion of vessels inaccessible to the ligature. Trans Am Surg Assoc. 29:389-410, 1911.

9. Cushing H. Electro-surgery as an aid to the removal of intracranial tumors. Surg Gynecol Obstet. 47:751-784, 1928.

10. Poppen JL. Prevention of postoperative extradural hematoma. Arch Neurol Psychiatry. 1935;34:1068-1069. 
11. Kim SH, Lee JH, Joo W, Chough CK, Park HK, Lee KJ, Rha HK. Analysis of the risk factors for development of post-operative extradural hematoma after intracranial surgery. Br J Neurosurg. 2015;29:243-248.

12. Lillemäe K, Järviö JA, Silvasti-Lundell MK, Antinheimo JJ-P, Hernesniemi JA, Niemi TT. Incidence of postoperative hematomas requiring surgical treatment in neurosurgery: a retrospective observational study. World Neurosurg. 2017;108:491-497.

13. Sinar EJ, Lindsay KW. Distant extradural haematoma complicating removal of frontal tumours. J Neurol Neurosurg Psychiatry. 1986;49:442-444.

14. Jeon JS, Chang IB, Cho BM, Lee HK, Hong SK, Oh SM. Immediate postoperative epidural hematomas adjacent to the craniotomy site. J Korean Neurosurg Soc. 2006;39:335-339.

15. Fukamachi A, Koizumi H, Nagaseki Y, Nukui H. Postoperative extradural hematomas: computed tomographic survey of 1105 intracranial operations. Neurosurgery. 1986;19:589-593.

16. Jha AN, Morris K. Iatrogenic ex vacuo subdural hygroma: and a complication of the modified “Poppen” suture. J Neurol Neurosurg Psychiatry. 1986;49:1084-1085.

17. Onodera H, Furuya Y, Uchida M, Nakayama H, Nakamura H, Sakakibara Y, Taguchi Y. Intracranial foreign body granuloma caused by dural tenting suture. Br J Neurosurg. 2011;25:652-654.

18. Nishiyama Y, Kanemaru K, Yoshioka H, Hanihara M, Horikoshi T, Kniouchi H. Intracranial pial arteriovenous fistula caused by dural tenting: case report. Neurol Med Chir (Tokyo). 2014;54:242-244.

251 19. Swayne OB, Horner BM, Dorward NL. The hitch stitch: an obsolete neurosurgical technique? Br J Neurosurg. 2002;16:541-544; discussion 544. 
254 21. Przepiórka Ł, Kunert P, Żyłkowski J, Fortuniak J, Larysz P, Szczepanek D, Wiśniewski

255 K, Jaskólski D, Ładziński P, Rola R, Trojanowski T, Marchel A. Necessity of dural

256 tenting sutures in modern neurosurgery: protocol for a systematic review. BMJ Open.

$257 \quad$ 2019;9:e027904.

258 22. Winston KR. Dural tenting sutures in pediatric neurosurgery. Pediatr Neurosurg.

$259 \quad 1998 ; 28: 230-235$. 
260 Figure 1: Acute subdural hematoma caused by a dural needle (patient who needed 261 reoperation). 
262 Figure 2: A safe gap between the cortex and the dura is visible. 
263 Figure 3: Schematic illustration of the dural tenting suture technique during postsurgical 264 closure. Note that the needle does not traverse the full dural thickness, and this can be 265 achieved by clear visualization of the cortical side of the dura. 
Table 1: Preoperative patient information

\begin{tabular}{lccc}
\multirow{2}{*}{\multicolumn{1}{c}{ Patient Characteristics }} & Group 1 & Group 2 & Group 3 \\
\cline { 2 - 4 } & $71(48.6)$ & $67(45.9)$ & $65(44.8)$ \\
\hline Intrinsic tumor & $64(43.8)$ & $69(47.3)$ & $67(46.2)$ \\
Extrinsic tumor & $11(7.5)$ & $10(6.8)$ & $13(9.0)$ \\
Vascular lesion & $0(0.0)$ & $0(0.0)$ & $93(64.1)$ \\
Second surgery & $51(34.9)$ & $55(37.7)$ & $54(37.2)$ \\
Preoperative midline shift $>1 \mathrm{~cm}$ & & & \\
\hline
\end{tabular}


Table 2: Postoperative Complications

\begin{tabular}{l|ccc}
\hline \multicolumn{1}{c}{ Complication } & Group 1* & Group 2 & Group 3 \\
\cline { 2 - 4 } & $3(2.1)$ & $0(0.0)$ & $0(0.0)$ \\
\hline $\begin{array}{l}\text { Contusion } \\
\text { Acute subdural hematoma }\end{array}$ & $2(1.4)$ & $0(0.0)$ & $0(0.0)$ \\
Epidural collection & & & \\
$\quad \leq 3 \mathrm{~mm}$ & $6(4.1)$ & $5(3.4)$ & $7(4.8)$ \\
$>3 \mathrm{~mm}$ & $3(2.1)$ & $4(2.7)$ & $3(2.1)$ \\
$\begin{array}{l}\text { CSF accumulation } \\
\text { Reoperation due to hematoma }\end{array}$ & $7(4.8)$ & $5(3.4)$ & $5(3.4)$ \\
$\begin{array}{l}\text { Subdural hygroma (CT imaging } \\
\text { first month after surgery) }\end{array}$ & $1(0.7)$ & $0(0.0)$ & $0(0.0)$ \\
& & & $4(2.8)$ \\
\hline
\end{tabular}

* Patients in group 1 had more complications than those in the other 2 groups, although this difference is not statistically significant. 


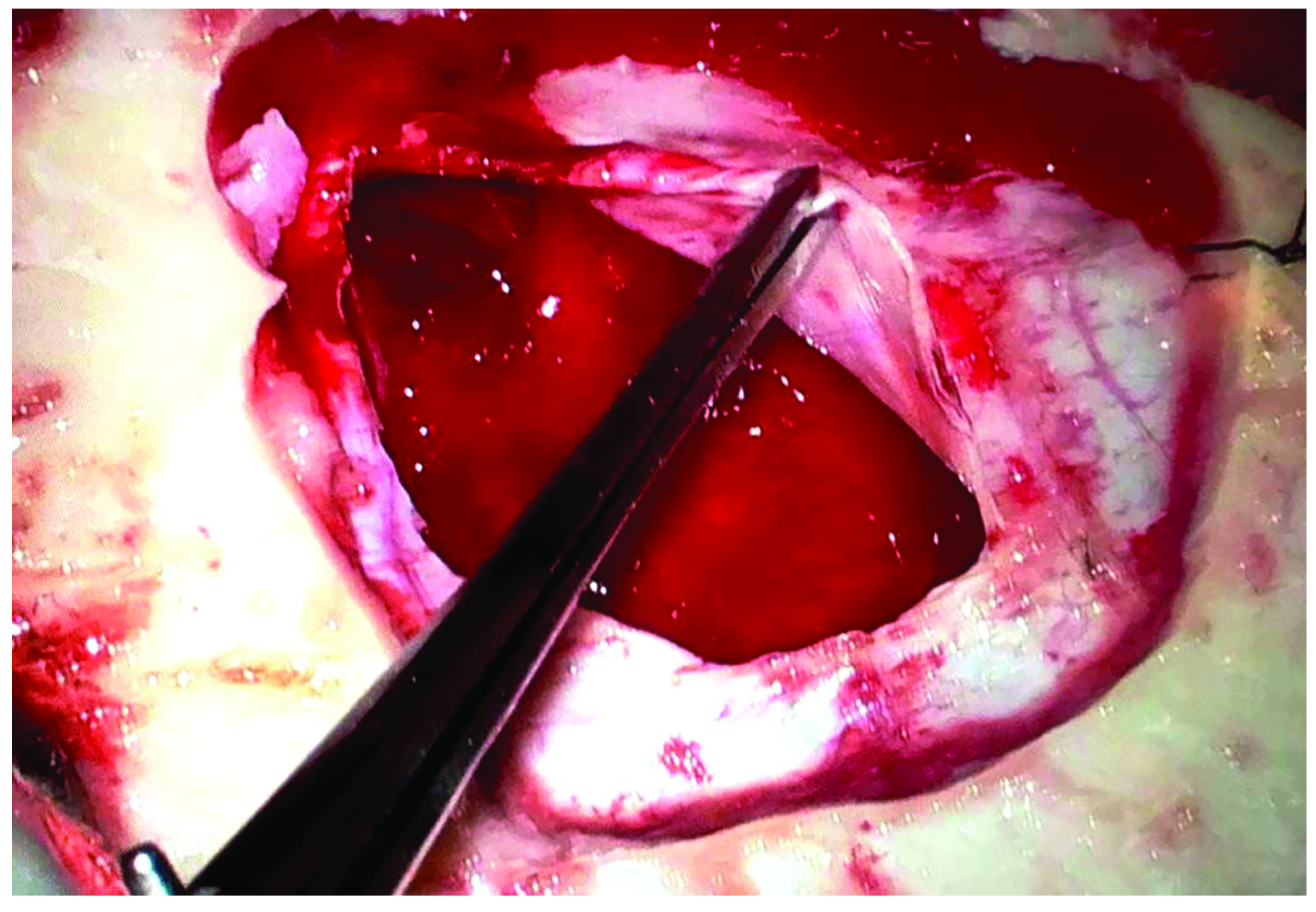




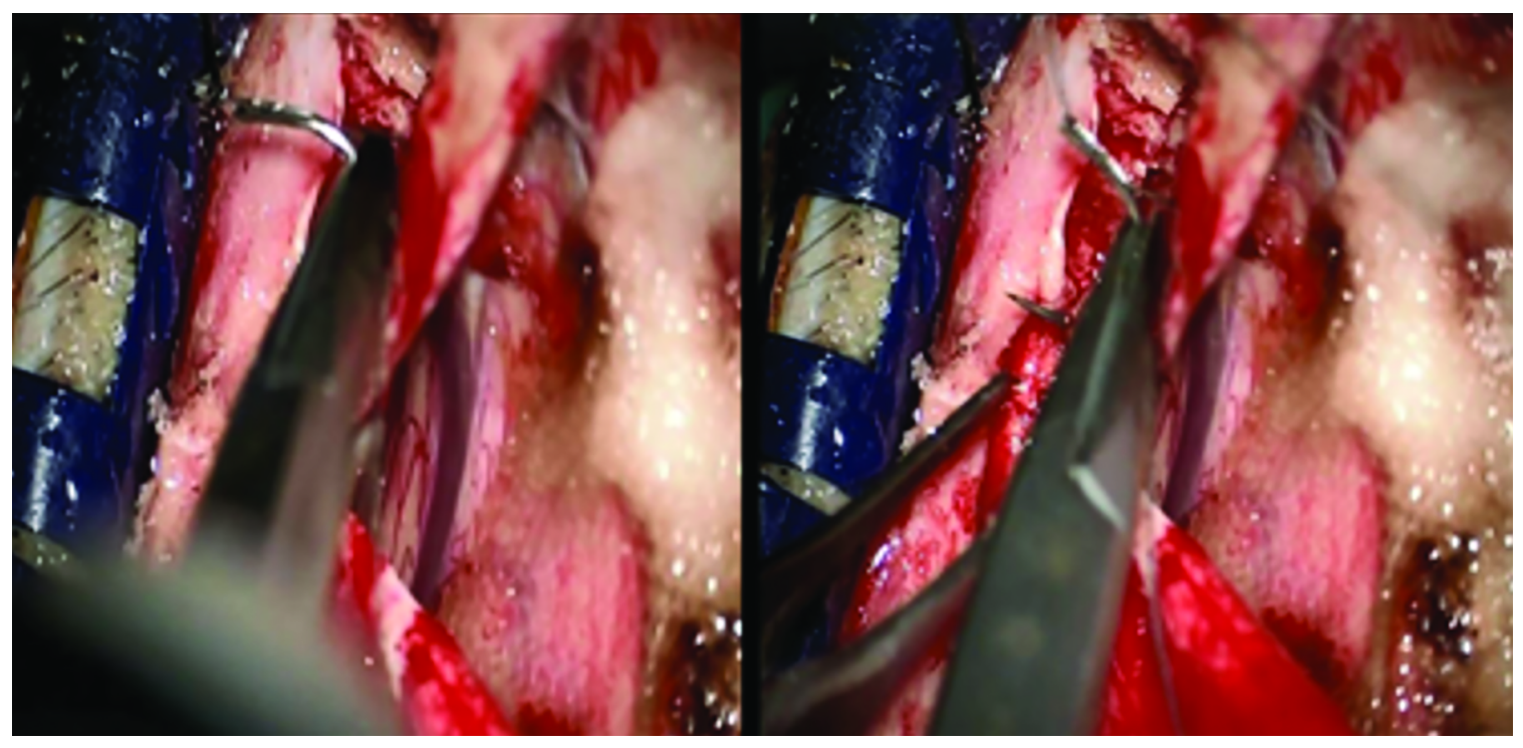




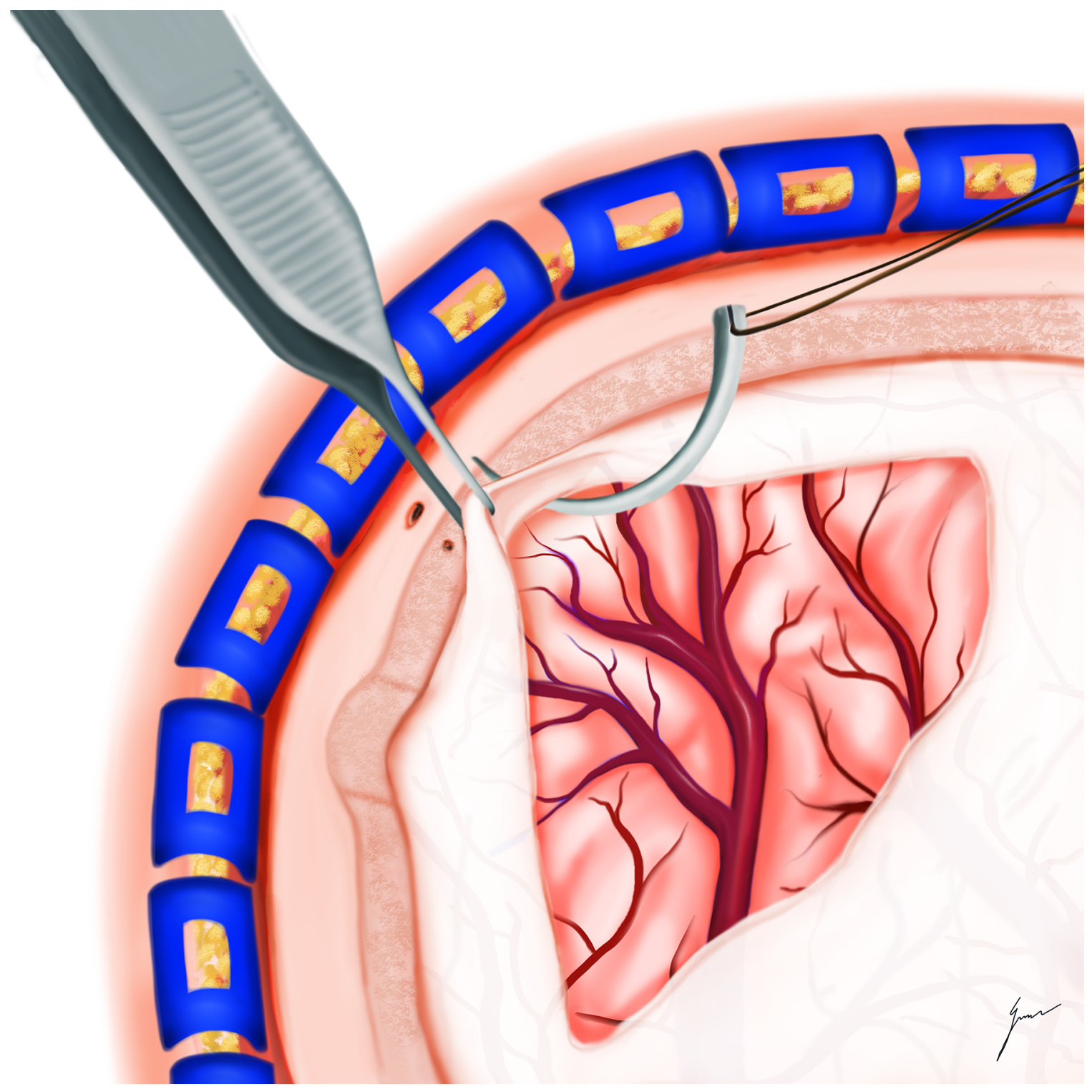


Is Placing Prophylactic Dural Tenting Sutures a Dogma?

Abbreviations: CSF, cerebrospinal fluid; CT, computed tomography; EDH, epidural hematoma 